\title{
Abnormal origin of left ovarian artery from the inferior renal polar artery - A rare anatomical variation
}

\author{
Eranga URR ${ }^{1}$, Samarawickrama MB ${ }^{1}$, Rodrigo $\mathrm{M}^{1}$, Nanayakkara $\mathrm{PGCL}^{1}$ \\ ${ }^{1}$ Department of Anatomy, Faculty of Medicine, University of Ruhuna, Sri Lanka
}

\begin{abstract}
Anatomical variations of the renal arteries and gonadal arteries are not uncommon owing to their embryological origin. Awareness of possible anatomical variations of the renal artery and gonadal artery is essential for surgeons who perform open and minimally invasive procedures of the kidneys and retroperitoneal region to prevent disastrous haemorrhagic complications during those procedures and for radiologists to prevent misinterpretations of imaging and to prevent vascular complications during interventional procedures.
\end{abstract}

The present report describes an unusual variation of the left ovarian artery arising from the inferior renal polar artery which originated from the abdominal aorta superior to the main renal artery.

Key words: Ovarian artery, inferior renal polar artery

\section{Introduction}

The renal arteries are a set of paired branches of the abdominal aorta arising just below the origin of the superior mesenteric artery ${ }^{1}$. Both renal arteries cross the corresponding crus of the diaphragm at right angles to the aorta and enter the renal hilum in between the renal vein and the ureter at the level of the first lumbar vertebra. The right renal artery is longer and often higher than the left. Although a single renal artery supplying each kidney is classically described, it is subject to anatomical variations frequently ${ }^{2}$. Knowledge of these anatomical variations of the renal vasculature is of great importance with the advent of vascular reconstructive surgeries, renal transplants, and various radiological and urological procedures carried out in this region $^{2}$.

The renal arteries themselves may vary in their level of origin, calibre and obliquity. They give off branches to suprarenal gland, ureter, renal capsule, perinephric tissue and the renal pelvis in their extrarenal course ${ }^{1}$. Near the renal hilum, each renal artery divides into anterior and posterior divisions, which in turn divide into segmental arteries supplying the renal segments. Accessory renal arteries which are believed to be derived from persistent embryonic lateral splanchnic arteries are not uncommon findings ${ }^{1,2}$. They can be found in more than $30 \%$ of the population and usually arise from the aorta below (less commonly above) the main renal artery and follow it to the renal hilum ${ }^{1}$.

The gonadal arteries arise from the abdominal aorta a just inferior to the renal arteries at the level of the second lumbar vertebra. Then they pass inferolaterally under the parietal 
Eranga URR, Samarawickrama MB, Rodrigo M, Nanayakkara PGCL - Abnormal origin of left ovarian artery from the inferior renal polar artery - A rare anatomical variation

peritoneum on psoas major muscle ${ }^{1}$. However, anomalous origins, course and numerical variations of the gonadal arteries are reported in the literature. Gonadal arteries are reported to be originated from renal arteries, accessory renal arteries, suprarenal artery, lumbar arteries, common iliac artery and superior epigastric artery ${ }^{3}$. Gonadal arterial anatomy and its anomalies are widely being studied because of their importance in testicular and renal surgeries. M Shoja et al have reported that aberrant gonadal arteries are associated with accessory renal arteries ${ }^{4}$. Although these variations can be attributed to its embryonic development, the exact embryonic signals which govern the formation of accessory renal arteries or aberrant gonadal arteries remain unknown ${ }^{4}$.

\section{Case report}

We have encountered an anomalous origin of a left ovarian artery from an inferior renal polar artery during routine educational dissections of the cadavers in the Department of Anatomy, Faculty of Medicine, University of Ruhuna, Sri Lanka.

We observed an accessory renal artery which originates $1 \mathrm{~cm}$ above the origin of the left renal artery passing to the inferior pole of the left kidney during dissection of the abdomen of a 70-year-old female cadaver. This artery was identified as the inferior renal polar artery. The artery passes downwards towards the left renal pole crossing the left suprarenal vein, left renal vein, branches of the left renal artery and the left ureter anteriorly. The left ovarian artery was observed to originate from the inferior renal polar artery about $3 \mathrm{~cm}$ proximal to its entry to the renal substance. Two tributaries of the left renal vein were observed draining blood from the renal hilum and the left ovarian vein was found to be draining to the inferior of the two (Fig 1).

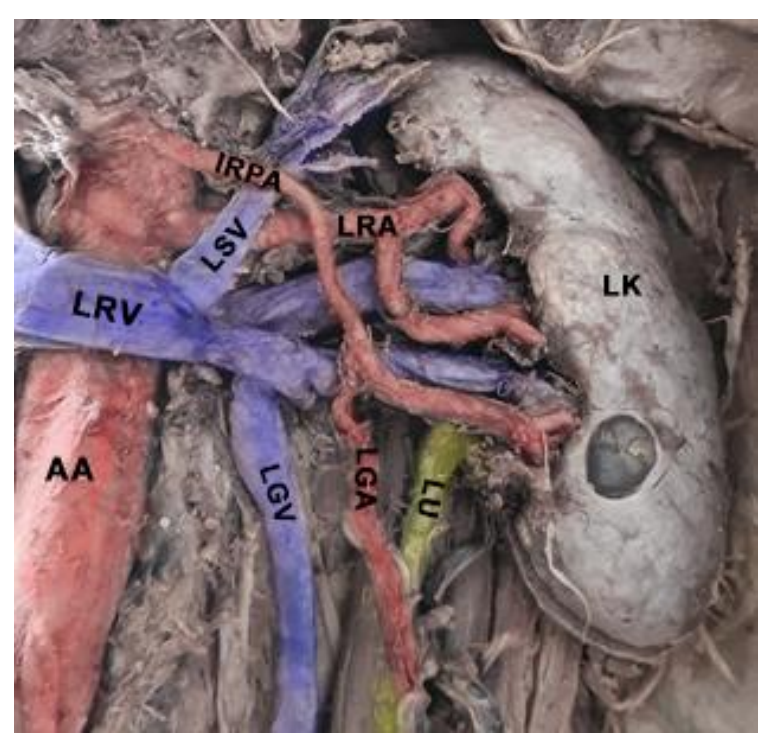

Figure 1 - Left retroperitoneal space showing, inferior renal polar artery with a high origin associated with an anomalous origin of ovarian artery from it.

(LK - left kidney, LU - left ureter, AAabdominal aorta, LRA - left renal artery, IRPA - Inferior renal polar artery, LGV - left gonadal artery, LRV - left renal vein, LSV left suprarenal vein, LGV - left gonadal vein)

On the right side, the normal distribution of the renal artery was observed and the ovarian artery had its usual origin from the abdominal aorta below it. Four venous tributaries were found at a distance of $2.5 \mathrm{~cm}$ from the renal hilum on the right side and the right ovarian vein was observed to be draining to the inferior vena cava. 
Eranga URR, Samarawickrama MB, Rodrigo M, Nanayakkara PGCL - Abnormal origin of left ovarian artery from the inferior renal polar artery - A rare anatomical variation

\section{Discussion}

Although variations of the renal arteries are not uncommon, a high origin inferior renal polar artery from the abdominal aorta associated with anomalous origin of a gonadal artery is rarely reported in literature 5,6. Awareness of these potential anatomical variations is of great importance for interventional radiologists, urologists, vascular and transplant surgeons who carryout open and minimal access surgical procedures in this region.

Accessory renal arteries to the inferior pole of the kidney crossing anterior to the ureter as in our case, may cause ureteric obstruction leading to hydronephrosis5. Awareness of these variations in the origin of these vessels highlights a potential importance for preprocedural imaging of the vasculature of the renal hilum to avoid complications during the surgery.

\section{Acknowledgement}

None

\section{Conflict of interest}

None

\section{Corresponding author}

Professor M B Samarawickrama MBBS (Ruh.) MS (Surg. Col.),

Professor in Anatomy and Consultant in General Surgery,
Department of Anatomy,

Faculty of Medicine, University of Ruhuna, Karapitiya, Galle, Sri Lanka.

E-mail: samaramb@gmail.com

CC BY 4.0

This is an Open Access article distributed under the terms of the Creative Commons Attribution 4.0 license (unless stated otherwise) which permits unrestricted use, distribution and reproduction in any medium, provided the original work is properly cited. Copyright is retained by the author(s).

\section{References}

1. Tunstall R. Grays Anatomy $41^{\text {st }}$ Edition.; 2016.

2. Mohammed AMA, Abdalrasol RGE, Abdalhai KA, Hamad MG. Accessory renal vessels. Acta Inform Medica. 2012;20(3):196-197. doi:10.5455/aim.2012.20.196-197

3. Acar HI, Yazar F, Ozan H. Unusual origin and course of the testicular arteries. Surg Radiol Anat. 2007;29(7):601-603. doi:10.1007/s00276-007-0232-2

4. Shoja MM, Tubbs RS, Shakeri AB, Oakes WJ. Origins of the gonadal artery: Embryologic implications. Clin Anat. 2007;20(4):428-432. doi:https://doi.org/10.1002/ca.20438

5. Ravery V, Cussenot O, Desgrandchamps $\mathrm{F}$, et al. Variations in arterial blood supply and the risk of hemorrhage during 
Eranga URR, Samarawickrama MB, Rodrigo M, Nanayakkara PGCL - Abnormal origin of left ovarian artery from the inferior renal polar artery - A rare anatomical variation

percutaneous treatment of lesions of the pelviureteral junction obstruction: report of a case of testicular artery arising from an inferior polar renal artery. Surg Radiol Anat. 1993;15(4):355-359.

doi:10.1007/BF01627892
6. Cho Y, Yoon S-P. Bilateral inferior renal polar arteries with a high origin from the abdominal aorta. Folia Morphol (Warsz). Published online April 2020. doi:10.5603/FM.a2020.0040 